\title{
Relevancia para el tratamiento de la sintomatología depresiva de los pacientes adictos
}

\author{
Sanabria, M.A.*; Díaz, O.**; Varela, J.***; Braña, T.**** \\ * Doctor en Psicología. Colaborador del "Programa para la Rehabilitación de Drogodependientes PROYECTO HOMBRE GALICIA". \\ * * Profesora Titular de "Técnicas Terapéuticas del Comportamiento". Facultad de Psicología. Universidad de Santiago. \\ *** Profesor Titular del Departamento de Métodos y Técnicas de Investigación en Ciencias del Comportamiento. Facultad de Psicología. Universidad de Santiago. \\ *** Profesora Titular del Departamento de Métodos. Facultad de Psicología. Universidad de Santiago.

\section{RESUMEN}

Muchos de los pacientes que acuden a los tratamientos por consumo de drogas acusan una serie de problemas solapados, a veces, por la propia adicción y que llegan a condicionar el resultado final de la intervención terapéutica.

Para precisar la incidencia de algunos de esos problemas estudiamos una muestra de 210 sujetos adictos sometidos a tratamiento ( $83 \%$ varones y $17 \%$ mujeres) tratando de interrelacionar diferentes tipos de problemas (legales, laborales, económicos, familiares, psicopatológicos, etc.) con su evolución terapéutica. Una de las variables que con más consistencia parece predecir los resultados del tratamiento es precisamente el nivel de sintomatología depresiva aducido por el propio paciente. Para valorar más la incidencia de esta variable fueron comparados los pacientes con sintomatología depresiva (75 sujetos) con los carentes de esos síntomas (135 sujetos). Los datos indican que los pacientes con sintomatología depresiva tienen un ambiente familiar más problemático y conflictivo, diferenciándose de manera significativa de aquellos otros carentes de dichos síntomas, lo que parece añadir más complicaciones a su recuperación.

A juzgar por estos datos, sería conveniente diversificar los tratamientos con intervenciones expresamente dirigidas al abordaje de la sintomatología depresiva y problemática familiar de cara a optimizar los resultados de la intervención.

Palabras Claves: Adicciones, sintomatología depresiva, resultados, tratamiento.

\section{SUMMARY}

Many of the patients who undertake treatment for drug consumption reveal a series of overlapping problems, sometimes due to their own addiction. These problems influence the final results of therapeutic supervision.

In order to accurately determine the influence of some of these problems, we studied a sample of 210 addicts subjected to treatment ( $83 \%$ males and $17 \%$ females), trying to correlate the various types of problems (legal, vocational, economic, psychopathologic as well as family problems, etc.) with their therapeutic evolution.

One of the variables which seems most consistently to predict treatment results is precisely the level of depressive symptomatology shown by the patient. To more accurately assess the influence of this variable, we compared 75 subjectpatients, all of them presenting depressive symptomatology, with 135 patients who did not. The data indicated that patients with certain levels of depressive symptomatology came from more problematic and conflictive family environments, a significant difference from those who did not present those symptoms. This seems to complicate their recovery.

Judging by the data, it would be advisable to diversify treatment, with procedures that specifically address depressive symptomatology and family environment, with the aim of optimizing treatment results.

Key words: Adictions, depressive symptomatology, results, treatment.

\section{INTRODUCCIÓN}

E relativamente frecuente encontrarse en pacientes que solicitan tratamiento por consumo de drogas con otra problemática psicopatológica que convive con la adicción. Este problema, al que no siempre se le ha prestado la debida atención, comienza a ser tenido en cuenta tanto en el campo terapéutico como en el de investigación, siendo dos las razones principales. La primera, de índole estadística, ya que más de la mitad de la población adicta tiene algún tipo de sintomatología psiquiátrica, aunque en ciertos casos se trata de alteraciones leves que se manifiestan como cambios en el estado de humor, estrés o ansiedad y que en buena medida se ven solapados por el consumo de las drogas. 
La segunda, hace referencia a la necesidad de prestarle atención a esta problemática debido a las interferencias que puede ocasionar en los tratamientos. El historial diagnóstico del paciente podría aclarar si el consumo de drogas es anterior a la problemática psicopatológica y cuál podría ser el peso de la adicción el la génesis de la psicopatología, o si más bien es posterior, en cuyo caso no sería descabellado pensar que la adicción pudiera tener una función compensatoria e incluso reparadora.

Las referencias de diferentes estudios de investigación destacan que las alteraciones psicopatológicas en población toxicómana suelen ser, en general, altas (Rounsaville, Weissman, Kleber y Wilber, 1983; Khantzian yTreece, 1985; Stoffelmayr, Benishek, Humphreys, Lee y Mavis, 1989; Casas, 1992; Pérez de los Cobos y Casas, 1993; Maisto, 1999; Pettinati, 2000).

Clerici, Rapolla y Castiglioni (1997) Ilegaron a diagnosticar al $90 \%$ de los 110 sujetos de su muestra con algún problema psiquiátrico. Arias, López-Ibor y Ochoa (1997) señalan que la prevalencia de los trastornos psiquiátricos en población adicta puede oscilar entre un 50-90\%.

Con bastante frecuencia muchos pacientes adictos suelen tener asociado algún otro problema de índole psicopatológico, figurando entre los más frecuentes la depresión, personalidad antisocial, alcoholismo y trastornos de ansiedad (Croughan, Miller, Wagelin, Whitman, 1982; Woody, McLellan y O'Brien, 1990; Calafat y Amengual, 1991; González, 1997; Cuadrado, 2000; Torneatto, 2000).

Los datos referidos a la evolución de algunos de estos trastornos suele parecer confusa, así por ejemplo las tasas referentes a la depresión ofrecen datos muy dispersos; tal dispersión guarda relación con el momento de la evaluación, con la dosis de droga consumida, con la solicitud o no de tratamiento. Así y todo, la sintomatología depresiva en estos pacientes ofrece tasas que oscilan entre el 20-60\%, reduciéndose durante los tratamientos a porcentajes del $17 \%$ y aumentando si vuelven a consumir (Dackis y Gold, 1984). En la Tabla 1 se ofrecen una serie de datos sacados de diferentes estudios que nos muestran la incidencia de este problema.

\section{TABLA 1. Sintomatología Depresiva}

\begin{tabular}{|c|l|c|}
\hline$\%$ & \multicolumn{1}{|c|}{ REFERENCIAS } & AÑO \\
\hline \multirow{2}{*}{46} & Hubbard, Rachal, Craddock y Cavanaugh & 1984 \\
60 & Dackis y Gold & 1984 \\
17 & Khantizian y Treece & 1985 \\
$20-60$ & Stoffelmayr, Benishek, Humphreys y col & 1989 \\
$50-60$ & Woody, McLellan y O'Brien & 1990 \\
21 & Arias, López-lbor y Ochoa & 1997 \\
\hline
\end{tabular}

Considerando los datos de la Tabla 1, cabe pensar que los problemas depresivos son un hándicap añadido a la drogadicción ya que, por un lado, estos problemas afectan, por lo menos, a la mitad de los pacientes adictos $y$, por otro, es uno de los trastornos que con más frecuencia convive con la drogadicción (Cox, Norton, Dorward y Fergusson, 1989; Pérez de los Cobos, 1996; Blume, 1999).

La presencia de esta problemática tiene importantes implicaciones para el tratamiento, afectando a la respuesta, a la duración y a los resultados del mismo; de manera que comenzar un tratamiento con un problema psicopatológico añadido augura peores resultados (McLellan, Luborsky, Woody, O'Brien y Druley, 1983; Svanum y McAdoo, 1989; Clopton, Weddige, Contreras, Fliszer y Arredondo, 1993; Szerman y Delgado, 1994; McMahon, 1999; Moggi, 1999; Brienza, 2000).

\section{OBJETIVOS}

Dada la asociación de la sintomatología depresiva con la toxicomanía vamos a tratar de valorar la influencia de este problema en la evolución y resultados del tratamiento, para ello nos hemos planteado los siguientes objetivos:

$1^{\circ}$. Valorar la influencia que la sintomatología depresiva puede tener en diferentes áreas, especialmente la familiar, por sus implicaciones en la recuperación y evolución de los pacientes adictos sometidos a tratamiento.

$2^{\circ}$. Comparar y valorar los resultados del tratamiento en función de la sintomatología depresiva aducida por los propios pacientes, tratando de clarificar el valor pronóstico de la misma.

\section{MÉTODO}

Para la valoración de estos aspectos hemos adoptado un diseño longitudinal de panel, ya que la misma muestra seleccionada es seguida y valorada en diferentes momentos y a lo largo de todo el período de seguimiento que abarca un año y medio, repitiendo las medidas de evaluación a intevalos de 6 meses.

\subsection{Instrumentos}

Para la recogida de datos hemos elaborado nuestra Entrevista Estructurada de Recogida de Datos (EERD), se trata de una adaptación, con algunas modificaciones, de la utilizada en el proyecto EMETYS (Sánchez-Carbonell, Brigós y Camí, 1989), inspirada a su vez en el ASI (Addiction Severity Index) (Mclellan, Luborsky, Cacciola, Griffith, Evans, Barr y O’Brien, 1985).

El instrumento se aplica de manera progresiva, precisando con preguntas que tienden a medir el número, la extensión y la duración de las diferentes áreas, tales como consumo y abstinencia de drogas, situación laboral, delictivo-judicial, etc., abarcando desde el pasado 
hasta los 30 últimos días, considerando en este caso, la referencia de la situación del problema en el momento actual.

Para la patología depresiva se incluyó un apartado en el cuestionario que recoge si el paciente acude al centro con diagnóstico y tratamiento para este cuadro, si el diagnóstico lo ha realizado el equipo terapéutico de drogodependencias en el momento del ingreso o si el paciente presenta una serie de síntomas claramente depresivos según criterios del DSM III-R. Estos síntomas hacen referencia a la pérdida de interés o motivación en tareas habituales, sentimientos de inutilidad, autorreproche o culpa, dificultades para la concentración, pensamientos de muerte, ideación suicida y finalmente, intentos de suicidio en los 30 últimos días.

En la valoración de las relaciones familiares utilizamos la escala FES (TEA, 1984) que valora el clima familiar o nivel de adaptación existente en el seno de la familia a través de las subescalas de la cohesión o grado de compenetración y ayuda mutua, la expresividad de sentimientos e ideas y los conflictos.

En definitiva, los diferentes instrumentos empleados nos permitieron obtener información sobre:

A). El Índice Global de Adaptación (IGA) que resume en una puntuación que oscila entre 0 y 4 la situación evolutivo-terapéutica del paciente, correspondiendo a mayor puntuación mejores niveles de adaptación y evolución terapéutica. Al sujeto se le suma 1 punto por cada una de las condiciones establecidas que cumpla, a saber: no consumir heroína o droga principal objeto de la intervención, en segundo lugar, no consumir otras drogas o alcohol por encima de los 75 cc. alcohol puro/día, en tercer lugar, trabajar o estar matriculado en algún curso en los 3 últimos meses y, finalmente, no haber cometido actividad ilegal en los últimos 3 meses.

B). Otros problemas: los problemas considerados son los que pueden tener cierta relación con el consumo, tales como los de carácter delictivo-judicial, laboral, económicos y familiares. Se valora la existencia o no (dos niveles) de estos problemas según la estimación del propio paciente y referidos al último mes.
C). Relaciones familiares: estimadas en función del grado de cohesión o apoyo familiar, espontaneidad en la expresión de sentimientos e ideas y los conflictos, cada una de estas 3 variables puede alcanzar una puntuación que oscila entre 0 y 9.

Por otro lado hemos establecido dos niveles para la variable sintomatología depresiva, el primer nivel hace referencia a los pacientes carentes de dicha sintomatología, en el segundo se incluye a los pacientes que reunían cuatro o más síntomas de carácter depresivo.

Para valorar de manera más objetiva la abstinencia se tuvieron en cuenta los análisis de orina que algunos centros llevaban a cabo, pero no se pudo contar con los análisis de orina para la totalidad de la muestra. Por diversas razones este procedimiento no está generalizado en todos los centros, ni en todos los tratamientos, siendo más frecuente en Mantenimiento con Metadona pero no en otros. Algunos pacientes son reticentes a las pruebas, finalmente, cuando el paciente se desliga del centro (seguimiento) resulta imposible realizar esta prueba objetiva por la falta de medios y costes.

\subsection{Muestra}

La muestra total la integran 210 sujetos adictos $183 \%$ hombres $17 \%$ mujeres), se trata de una muestra representativa de la totalidad de los pacientes adictos implicados en tratamiento por consumo de drogas y estimada en función de los datos estadísticos del año anterior. Los pacientes muestreados corresponden a 5 centros de la Red Asistencial de Drogodependencias de la Comunidad Autónoma de Galicia que consintieron participar en el estudio. La media de edad de la muestra es de 25 años con un rango de 17 a 35 años.

En el momento de acudir a tratamiento la totalidad de la muestra son consumidores habituales de diversas sustancias, siendo el policonsumo el perfil característico para la práctica totalidad de la muestra, solamente el $2 \%$ de los pacientes quedarían excluidos de dicho perfil. El patrón de policonsumo se caracteriza por consumir cualquier sustancia a la que tengan acceso y al margen de un consumo especializado en un grupo de sustancias en concreto. Este patrón se puede apreciar en la Tabla 2, donde se recoge el historial adictivo de la muestra.

TABLA 2. Historial del Consumo de drogas

\begin{tabular}{|l|c|c|c|c|c|c|c|}
\hline \multicolumn{1}{|c|}{ SUSTANCIA } & $\begin{array}{c}\text { PROBADA \% } \\
\text { CONSUMO }\end{array}$ & $\begin{array}{c}\bar{x} \text { EDAD 1er } \\
\text { CONSUMO }\end{array}$ & $\begin{array}{c}\text { EDAD CONS. } \\
\text { REGULAR }\end{array}$ & $\begin{array}{c}\text { ORDEN de 1er } \\
\text { CONSUMO }\end{array}$ & \multicolumn{2}{|c|}{$\begin{array}{c}\text { CONSUMO ÚLTIMO MES } \\
\text { ABSTINENTES CONSUMIDORES }\end{array}$} & $\begin{array}{c}\text { NIVEL DE } \\
\text { CONSUMO MEDI0 }\end{array}$ \\
\hline Alcohol & 99 & 13.7 & 15.4 & $2^{\text {a }}$ & $15 \%$ & $85 \%$ & $42 \mathrm{cc} . /$ día \\
Hachís & 99 & 15.4 & 16 & $3^{\mathrm{a}}$ & $47 \%$ & $53 \%$ & $1-2 \mathrm{por} \mathrm{semana}$ \\
Heroína & 98 & 19 & 20.3 & $6^{\circ}$ & $28 \%$ & $72 \%$ & $1-2$ al día \\
Cocaína & 98 & 18.6 & 20 & $5^{\circ}$ & $59 \%$ & $41 \%$ & 1 semanal \\
Alucinógenos & 70 & 17.8 & - & $7^{\circ}$ & $96 \%$ & $4 \%$ & $<1$ al mes \\
Tranquilizant. & 79 & 20.2 & 21.5 & $8^{\circ}$ & $53 \%$ & $47 \%$ & $1-3$ al mes \\
Anfetaminas & 68 & 17.5 & - & $4^{\circ}$ & $97 \%$ & $3 \%$ & $\leq 1$ por semana \\
\hline
\end{tabular}


Como se puede apreciar en la Tabla, la sustancia más consumida es el alcohol con un nivel de consumo medio que alcanza los 42 centímetros cúbicos de alcohol puro al día. De las sustancias ilegales la más consumida es la heroína, ya que el $72 \%$ de la muestra lo hace diariamente, seguida del hachís, tranquilizantes y cocaína consumidas de manera semanal por el 53\%, 47\% y $41 \%$ respectivamente, seguidas del resto de sustancias en menor escala.

Respecto al área psicopatológica, es la sintomatología depresiva la que acumula mayor incidencia, ya que el $41 \%$ de dichos pacientes confiesa haber tenido problemas de este tipo; el pensamiento suicida ha estado presente en el $32 \%$ de los casos en el pasado y un $8 \%$ dicen haberlo tenido en el presente. Los intentos de suicidio se elevan al $16 \%$ en el pasado y al $2 \%$ en el último mes. Un 18\% de los pacientes manifiestan que estos problemas les preocupan y afectan con cierta consideración e intensidad, estando dispuestos a recibir tratamiento para atajarlos, si bien ninguno de los pacientes estaba recibiendo tratamiento específico por problemas de carácter depresivo en el momento de la entrevista inicial.

\section{3.- Procedimiento}

Una vez que se contactó con las distintas Unidades Asistenciales y se les explicó el plan de trabajo, se elaboró un perfil prototipo del paciente en función de variables de carácter sociodemográfico y teniendo en cuenta los usurarios atendidos el año anterior, fijándose un número proporcional y representativo de pacientes a reclutar para el estudio.

Los pacientes fueron seleccionados al azar y a medida que acudían a los centros, siempre y cuando encajaran en el perfil establecido en función de los datos del año anterior. Se solicitaba su consentimiento para ser incluidos en el estudio y evaluarlos en diferentes momentos. Los contactos con los pacientes se realizaban a través del propio centro, cuando esta alternativa no era posible se acordaba con el paciente otro lugar alternativo (cafeterías, bibliotecas...) para realizar las entrevistas. Los contactos del seguimiento se efectuaban con independencia de que el sujeto se hubiese desligado del tratamiento, de ahí que en algunas ocasiones, ante la imposibilidad de contar con la presencia física del paciente, la entrevista hubo de realizarse por teléfono. Al final, por diferentes motivos (fallecimiento, cambio de domicilio, encarcelamiento, busca y captura, etc.) un total de 32 pacientes no pudieron ser entrevistados.

Los entrevistadores, uno por centro, eran licenciados en psicología y asistentes sociales a los que se les adiestró previamente; además, tuvieron una semana de rodaje en los centros para familiarizarse con los cuestionarios, sin que esas entrevistas fueran consideradas para el estudio.

\subsection{Tratamientos}

Los pacientes fueron asignados fundamentalmente a dos tipos de tratamiento: Programas Libres de Drogas y de Mantenimiento con Metadona. Dentro de los primeros había dos tipos de modalidades, una de carácter Ambulatorio, el paciente acude al centro a las sesiones de terapia y apoyo (generalmente gran parte de la mañana) pasando el resto de día en compañía de la familia o persona responsable; el Centro de Día, se caracteriza por una mayor estancia del paciente en el centro, compaginando la actividades terapéuticas con otras complementarias de carácter ocupacional, formativo y de ocio. El objetivo de estos tratamientos es conseguir la abstinencia del consumo de drogas y conseguir que el paciente pueda llevar un estilo de vida más adaptativo e integrado en su medio social.

En los tratamientos de Mantenimiento con Metadona la intervención gira entorno al uso de esa sustancia con fines terapéuticos, el paciente acude al centro a recibir su dosis y terapia de apoyo, tratando de implicarlo en otro tipo de actividades con la intencionalidad de que pueda acceder a programas Libres de Drogas. El objetivo es conseguir que el paciente no consuma la heroína adquirida en la calle desviando su consumo hacia la metadona administrada bajo control y seguimiento terapéutico, con la finalidad de que una vez conseguido esto el paciente pueda acceder a un programa libre de drogas, de cara intentar conseguir la abstinencia total.

Así pues y teniendo en cuenta que bastantes pacientes fueron alternando de modalidad terapéutica en ambos sentidos (de Mantenimiento de Drogas a Libres de Drogas y viceversa) y en función de su evolución, no vamos a analizar los resultados según el tipo de tratamiento por el solapamiento que existe en nuestra muestra, sino que vamos a considerar el evento terapéutico como criterio único con independencia de la modalidad.

\section{RESULTADOS}

Para determinar la posible incidencia de la sintomatología depresiva en otras áreas problemáticas, llevamos a cabo un primer análisis incluyendo los distintos tipos de problemas: de carácter legal, laborales, económicos y los relativos al ámbito familiar; todos ellos referidos al pase inicial o previo al tratamiento. Dado que la variable referida a "otros problemas" es dicotómica, se analizó el contraste mediante la prueba no paramétrica de Kolmogorov-Smirnov para dos muestras, utilizando como variable de agrupamiento la sintomatología psicopatológica. Los dos grupos de sujetos, con y sin indicios de depresión, fueron contrastados en los distintos tipos de problemas. Si bien los pacientes con síntomas depresivos en general presentan más problemas en varias de las áreas consideradas, la mayoría no llegan a alcanzar signi- 
ficatividad estadística; la única que parece diferenciar de manera significativa a los dos grupos es la familiar ( $Z=$ $1.47 ; p=0.02)$, por lo que tratamos de profundizar en el estudio de esta variable.

Tratando de concretar más la problemática familiar en los pacientes adictos, comparamos las puntuaciones de la escala FES, que mide la calidad de las relaciones en el ámbito familiar, tal como son percibidas por el hijo adicto al inicio del tratamiento. Se realizaron un total de 10 pruebas t de Student con las diferentes subescalas de la FES, siendo significativos los contrastes referidos a la cohesión, expresividad y conflicto, resultados que se presentan en la Tabla 3.

\section{TABLA 3. Comparación de Sujetos con/sin depresión y relaciones familiares}

\begin{tabular}{|l|c|c|c|c|c|c|}
\hline & $\mathbf{n}$ con. & $\mathbf{N} \sin$ & $\bar{x} \operatorname{con}$ & $\bar{x} \sin$ & $\mathbf{t}$ & $\mathbf{P}$ \\
\hline COHESION Inicio & 75 & 135 & 5.64 & 6.45 & $\mathbf{2 . 2 8}$ & 0.02 \\
\hline EXPRESIVIDAD Inicio & $"$ & $"$ & 4.84 & 5.36 & $\mathbf{1 . 9 5}$ & 0.04 \\
\hline CONFLICTO Inicio. & " & $"$ & 3.81 & 3.08 & $\mathbf{- 2 . 3 0}$ & 0.02 \\
\hline
\end{tabular}

De acuerdo con los resultados obtenidos, los pacientes depresivos tienen unas relaciones familiares más conflictivas; esta problemática se manifiesta en que existe menos compenetración, ayuda y apoyo entre los miembros del sistema familiar, más dificultades para expresar los sentimientos e ideas, mayores niveles de conflictividad en sus relaciones plasmados en riñas, disputas y peleas. Así pues, estos datos vienen a confirmar los supuestos de la primera hipótesis, que hacía referencia a un ambiente familiar más deteriorado en estos pacientes.

Para valorar la función pronostica atribuible a la sintomatología depresiva llevamos a cabo un Análisis de Regresión Múltiple, dicho análisis permite apreciar qué variables consideradas como independientes predicen y, por tanto influyen, en otra considerada como dependiente o a predecir.

Si consideramos el IGA de cada uno de los pases como VD o variable a predecir, la sintomatología depresiva entra en la ecuación de predicción con valor significativo. Ahora bien, como el IGA se construye a partir de los valores de otras variables y para evitar la linealidad, prescindimos de aquellas que forman parte del IGA. Las variables que tienen un valor predictivo significativo son, el número de días que el sujeto ha permanecido en tratamiento, el consumo bruto de alcohol y la sintomatología depresiva, con una capacidad predictiva sobre el IGA del $28 \%(R 2=0.28)$ en el primer caso (Tabla 4$)$ y del $22 \%$ $(\mathrm{R} 2=0.22)$ en el segundo (Tabla 5$)$. El signo negativo de las betas referentes a la sintomatología depresiva y consumo bruto de alcohol señalaría a los pacientes que en ausencia de sintomatología y poco o ningún consumo de alcohol tendrían una situación más adaptativa en base a mayores puntuaciones del IGA.
TABLA 4. Variables predictivas respecto al IGA final (Pase 6 meses)

\begin{tabular}{|c|c|c|c|c|c|}
\hline \multicolumn{1}{|c|}{ V.I } & B & S.E. B & Beta & T & Sig. T \\
\hline Sintomatología depresiva & $-\mathbf{0 . 3 2}$ & $\mathbf{0 . 0 9}$ & $\mathbf{- 0 . 2 9}$ & $\mathbf{- 3 . 3 0}$ & $\mathbf{0 . 0 0}$ \\
\hline Consumo bruto de alcohol & -2.00 & 6.58 & -0.28 & -3.07 & 0.00 \\
\hline N $^{0}$ de días en tratamiento & 0.24 & 0.06 & 0.32 & 3.58 & 0.00 \\
\hline (Constante) & 2.25 & 0.25 & & 9.02 & 0.00 \\
\hline
\end{tabular}

TABLA 5. Variables predictivas respecto al IGA final (Pase 12 meses)

\begin{tabular}{|c|c|c|c|c|c|}
\hline \multicolumn{1}{|c|}{ V.I } & B & S.E. B & Beta & T & Sig. T \\
\hline Sintomatología depresiva & $-\mathbf{0 . 5 0}$ & $\mathbf{0 . 1 4}$ & $\mathbf{- 0 . 3 2}$ & $\mathbf{- 3 . 4 6}$ & $\mathbf{0 . 0 0}$ \\
\hline Consumo bruto de alcohol & -2.00 & 7.00 & -0.29 & -3.15 & 0.02 \\
\hline N $^{0}$ de días en tratamiento & 0.24 & 0.98 & 0.29 & 2.52 & 0.01 \\
\hline (Constante) & 1.98 & 0.31 & & 6.23 & 0.00 \\
\hline
\end{tabular}

Estos análisis vienen a dar cierta consistencia a la hipótesis de que la sintomatología depresiva, junto con otras variables, marcan diferencias en la evolución y resultado final del tratamiento, resultados que van en la misma dirección de los obtenidos en otras investigaciones que apuntan a un peor pronóstico y evolución terapéutica en los pacientes afectados por algún grado de severidad psicopatológica (McLellan, Woody, Luborsky, O’Brien y Druley, 1983; San, Camí, Peri, Mata y Porta, 1989; Clopton, Weddige, Contreras, Fliszer y Arredondo, 1993; Bootsmiller, 1998; Zeitlin, 1999). Por otra parte, dentro de todo el conjunto de variables exploradas, esta tríada aparece como los mejores indicadores pronóstico de la evolución y resultados del tratamiento a medio y largo plazo.

Estos resultados también ponen de manifiesto que los pacientes adictos de nuestro estudio, tienen una peor evolución cuando presentan síntomas de carácter depresivo, han permanecido menos días en tratamiento y tienden a consumir más alcohol durante y después de la intervención; de manera que estos problemas suponen una dificultad añadida a la hora de erradicar el consumo de drogas e instaurar un estilo de vida más adaptativo en ellos. Por otra parte, resulta interesante tenerlas en consideración a la hora de tomar decisiones para mejorar la implementación de los programas y optimizar la eficacia de los mismos.

\section{DISCUSION}

En comparación con los resultados de otros estudios también los pacientes explorados en la sintomatología depresiva de nuestra muestra ofrecen, en el $41 \%$ de los casos, indicios evidentes de sufrir en algún grado ese tipo de problemas, porcentaje que se sitúa en la media apreciada en otros trabajos y que oscila entre el 20-60\%, 
como se puede apreciar en las referencias de la Tabla 1. De manera que podemos considerar nuestra muestra de adictos equiparable a las muestras exploradas en otros estudios en referencia a este tipo de problemas.

Es evidente que en la terapéutica por consumo de drogas nos encontramos con muchos pacientes que acuden a los programas con otros problemas de carácter psicológico añadidos o interconectados con la propia adicción. Al contrastar los problemas de carácter depresivo con otro tipo de problemáticas de diferentes áreas, sobresalen los del área familiar, siendo ésta la más afectada por la sintomatología de carácter depresivo que acumula el paciente adicto. En este sentido la falta de entendimiento con los miembros del sistema familiar al que pertenece el adicto es evidente, dificultando más, si cabe, unas relaciones ya de por si deterioradas que el paciente adicto mantiene con la familia de origen. La drogodependencia representa junto a la problemática depresiva un doble hándicap para la integración de estos pacientes en un clima familiar adecuado que facilite su recuperación.

El deterioro del clima familiar que parecen presentar los pacientes con sintomatología depresiva parece caracterizarse por la falte de entendimiento, empatía y enfrentamientos constantes entre los miembros del sistema familiar, lo cual añade más dificultades a la hora de recabar ayuda y apoyo de la familia, tan importante para su rehabilitación. Sería interesante poder planificar desde los propios programas algún tipo de intervención familiar para mejorar y optimizar las relaciones respecto al paciente adicto. Tal conveniencia sería una exigencia cuando la ayuda terapéutica de la familia sea imprescindible y forme parte del plan terapéutico.

Con bastante frecuencia este tipo de problemática adicional que acumulan los pacientes cuando acuden a los tratamientos, puede pasar desapercibida al estar solapada por el propio consumo de sustancias, sin embargo puede ser tan determinante como la propia adicción, llegando a condicionar la evolución terapéutica de estos pacientes, como parece deducirse de los resultados de nuestro estudio.

Así pues, es conveniente poder identificar algunos de estos problemas en convivencia con la propia adicción, determinar su alcance y precisar su influencia sobre el objetivo de la abstinencia. Lo ideal sería que todos los tratamientos pudieran prestar la atención que se merece la problemática diversa con que acuden los adictos a los tratamientos; lo cierto es que unas veces por falta de medios, de tiempo, de especialista y la inercia de los programas hace que muchos de esos problemas no sean tenidos en cuenta ni valorados en su justa medida.

Nuestro estudio ha pretendido poner de manifiesto que la propia información referida por el paciente y centrada en la sintomatología depresiva puede ser muy valiosa y útil, ayudando a identificar a un subgrupo de pacientes a los que se le deberá prestar más cuidados si queremos que su evolución terapéutica sea exitosa. Ello puede hacerse a pesar de que los programas no contemplen la aplicación estandarizada de instrumentos de diagnóstico y evaluación ni con especialistas dedicados al abordaje específico de la sintomatología depresiva. Pero si puede ayudar a identificar a los pacientes que necesitan más dedicación y atención terapéutica por parte de los equipos de tratamientos y educadores de los centros.

Efectivamente, entre las variables que con cierta significatividad estadística parecen predecir los resultados al final del seguimiento está la sintomatología depresiva aducida por el propio paciente que, junto con el consumo de alcohol y el número de días en tratamiento, alcanzan un cierto valor significativo en la ecuación de predicción explicando el $22 \%$ de la varianza, lo que da una idea de la influencia de estas variables en la consecución y estabilización de resultados satisfactorios al final del tratamiento.

Nuestros datos pueden ayudar a mejorar la planificación de las intervenciones con adictos. Lo ideal sería establecer un diagnóstico dual en pacientes con problemas psicológicos y de adicción para un mejor aprovechamiento de los recursos terapéuticos existentes, incluso en otras instituciones ajenas a los programas pero en estrecha colaboración con éstos, como pueden ser los servicios públicos de psiquiatría. Cuando no sea viable esta alternativa, es conveniente hacer notar a terapeutas y educadores que los pacientes con sintomatología depresiva requieren atenciones terapéuticas adicionales, de manera que la consecución de la abstinencia sea también un objetivo viable y alcanzable por ellos.

Estos cuidados pueden hacerse extensivos, ya no solo a la sintomatología depresiva, si no también al contexto familiar en el que vive el paciente, haciendo una valoración y si es preciso una intervención en el mismo, con la esperanza de que la mejora en las relaciones familiares redunde en la consecución de resultados más satisfactorios y estables para el propio paciente.

\section{REFERENCIAS BIBLIOGRAFICAS}

Arias, F., López-lbor, J. y Ochoa, E. (1997). Comorbilidad psiquiátrica en dependientes de opiáceos en tratamiento con naltrexona. Adicciones, 9, 235-253.

Blume, A.W. (1999). Neurocognitive dysfunction in duallydiagnosed patients: A potential roadblock to motivating behavior change. Journal of Psychoactive Drugs, 31, 111-115.

Bootsmiller, B.J. (1998). Methods of ensuring high followup rates: Lessons from a longitudinal study of dual diagnosed participants. Substance Use and Misuse. 33, 2665.2685.

Brienza, R.S., (2000). Depresión among needle exchange program and methadone maintenence clients. Journal of Substance Abuse Treatment, 18, 331-337. 
Calafat, A. y Amengual, M. (1991). Depresión, depresividad y toxicomanía. Adicciones, 3, 75-100.

Casas, M. (1992). Trastornos psíquicos en toxicomanías. Vol. 1. Sitges. Ediciones en Neurociencias.

Clerici, M., Rapolla, M.R. y Castiglioni, B. (1997). Trastornos por uso de drogas y comorbilidad psiquiátrica en un servicio para la toxicodependencia (SERT) durante un período de seis años. Adicciones, 9, 467-481.

Clopton, J.R., Weddige, R.L., Contreras, S., Fliszer, G. y Arredondo, R. (1993). Treatment outcome for substance misuse patients with personality disorder. International Journal of the Addictions, 28, 1147-1153.

Cox, B.J., Norton, G., Dorward, J. y Fergusson, P. (1989). The relationship between panic attacks and chemical dependencies. Addictive Behavior, 14, 53-60.

Croughan, J.L., Miller, J.P., Wagelin, D. y Whitman, B. (1982). Psychiatric illness in male an female narcotic addicts. Journal Clinical Psychiatry, 43, 225-228.

Cuadrado, P. (2000). Dependencia alcohólica con y sin trastornos psiquiátricos asociados. Adicciones, 12, 373.381.

Dackis, C.A. y Gold, M.S. (1984). Depression in opiate addicts: En S.M. MIRIN (Ed.). Substance abuse and psychopathology (pp. 19-40). Washington. American Psychiatric Press.

González, F. (1997). Patología dual. Proyecto, 24, 9-13.

Hubbard, R., Rachal, J., Craddock, S. y Cavanaugh, E. (1984). Treatment outcome prospective study (TOPS): Client characteristics and behaviors before, during and after treatment. En F. TIMS y J. LIDFORD (Eds.). Drug abuse treatment evaluation: Strategies, progress $y$ prospects. NIDA Research Monograph, 51. Washington. Rockville.

Khantzian, E. J. y Treece, C. (1985). DSM III psychiatric diagnosis of narcotic addicts. Recent finding. Archives General of the Psychiatry, 42, 1067-1071.

McMahon, R.C. (1999). Substance abuse history predicts depressión and relapse status among cocaine abusers. American Journal on Addictions, 8, 1-8.

Maisto, S.A. (1999). Methods of changing patterns of substance use among individuals with co-occurring schizophrenia and substance use disorder. Journal of Substance Abuse Treatment, 17, 221-227.

McLellan, A.T., Luborsky, L., Cacciola, J., Griffith, B.A., Evans, F., Barr, H.L. y O'Brien, CH. (1985). New data from the Addiction Severity Index. Reliability and validity in three centers. Journal Nervous and Mental Disease, 173, 7, 412-423.

McLellan, A.T., Luborsky, L., Woody, G., O'Brien,CH. y Druley, P.D. (1983). Predicting response to alcohol and drug abuse treatment. (Role of Psychiatric Severity). Archives General of the Psychiatry, 40, 620-625.

McLellan, A.T., Woody, G., Luborsky, L., O'Brien, CH. y Druley, K. (1983). Increased effectiveness of substance abuse treatment: A prospective study of patienttreatment "matching". Journal Nervous and Mental Disease, 171, 597-605.
Moggi, F (1999). Effectiveness of treatment for substance abuse and dependence for dual diagnosis patients: A model of treatment factors associated with one-year outcomes. Journal of Studies on Alcohol, 60, 856866.

Perez de los Cobos, J. y Casas, M. (1993). Dependencia de opiaceos y Psicopatología concomitante. En J. Cadafalch y M. Casas (Eds.). El paciente heroinómano en el hospital general. Bilbao. Ed.Plan Nacional de Drogas, CITRAN y Generalitat de Catalunya.

Perez de los Cobos, J. (1996). Depresiones aducidas (por el paciente) e inducidas (por las drogas) en las adicciones. Opinión, 5. 27-32

Pettinati, H.M. (2000). Gender and psychiatric comorbidity: impact on clinical presentation of alcohol dependence. American Journal on Addictions, 9, 242- 252.

Rounsaville, B.J., Weissman, M.M.; Kleber, H. y Wilber, $\mathrm{CH}$. (1983). Heterogeneity of psychiatric diagnosis in treated opiate addicts. Archives General of the Psychiatry, 39, 161-166.

San, L., Camí, J., Peri., Mata. R. y Porta, M. (1989). Success and failure at impatient heroin detoxification. British Journal of the Addiction, 84, 81-87.

Sánchez Carbonell, J., Brigós, B. y Camí, J. (1989). Evolución de una muestra de heroinómanos dos años después del inicio del tratamiento: Proyecto EMETYST. Medicina Clínica, 92, 135-139.

Stoffelmayr, B.E., Benishek, L.A., Humphreys, K., Lee, J. y Mavis, B. (1989). Substance abuse prognosis with an addictional psychiatric diagnosis: understanding the relationship. Journal of Psychoactive Drugs, 21, 145152.

Svanum, S y McAdoo, W. (1989). Predicting rapid rellapse following treatment for chemical dependence: $A$ marched-subjets design. Journal Consulting and Clinical Psychology, 57, 222-226.

Szerman, N. y Delgado, F. (1994). Diagnóstico dual, medicación y objetivos transicionales en el abordaje psicoterapéutico de las drogodependencias. Adicciones, 6, 5-14.

Tea (1984): Escalas de clima social: FES. Madrid. TEA Ediciones. (Ed. orig. 1981)

Torneatto, T. (2000). Diagnostic subgroups within a sample of comorbid substance abusers: correlates and characteristics. American Journal on Addictions, 9, 253-264.

Weiss. R.D. (2000). Utilization of psychosocial treatment by patients diagnosed with bipolar disorder and substance dependence. American Journal on Addictions, 9, 314-320.

Woody, G., McLellan, A.T. y O'Brien, CH. (1990). Research on psychopathology and addiction: Treatment implications. Special Issue: Research and policy. Drug and Alcohol Dependence, 24, 121-123.

Zeitlin, H. (1999). Psychiatric comorbidity with substance misuse in children and teenagers. Drug and Alcohol Dependence, 55, 225-234. 
